\title{
Argentina 2020: PANDEMia, DeUda eXterna y LA REgión
}

A LA DERECHA
Argentina 2020: the Pandemic, Foreign Debt and the Region's Turn to the Right

\section{FLORENCIA MONTAL}

Universidad Torcuato Di Tella, Argentina

\section{PATRICIO YAMIN VÁZQUEZ (1)}

Universidad de San Andrés, Argentina

\begin{abstract}
RESUMEN
Este artículo examina la coalición que sostiene al gobierno de Alberto Fernández en Argentina a la luz de la literatura de Ciencia Política sobre coaliciones y analiza los sucesos principales que tuvieron lugar en relación a tres grandes desafíos que el nuevo gobierno debió enfrentar durante su primer año: el manejo de la pandemia COVID-19, la renegociación de la deuda pública y la relación con los países de la región. En estas tres áreas se manifiesta, con variable intensidad, la diversidad de preferencias al interior de la coalición gobernante.
\end{abstract}

Palabras clave: Argentina, COVID-19, Deuda externa, coaliciones.

\begin{abstract}
This article examines the coalition supporting the government of Alberto Fernandez in Argentina against the Political Science literature on coalitions. It also analyzes major developments and trends in relation to three challenges before the new administration during its first year: the management of the COVID-19 pandemic, foreign debt renegotiations, and its relationship with neighbouring countries. These three areas show, with varying degrees of intensity, the diversity of preferences within the ruling coalition.
\end{abstract}

Keywords: Argentina, COVID-19, Foreign Debt, Coalitions. 


\section{INTRODUCCIÓN}

El 10 de diciembre de 2019 el peronismo volvió a gobernar Argentina. A diferencia de la última administración peronista, esta llegaba al poder en la forma de una coalición amplia con Alberto Fernández (AF) como presidente y Cristina Férnandez $(\mathrm{CF})$ como vicepresidenta. En esta contribución al Anuario de la Revista de Ciencia Política examinamos, en primer lugar, la actual coalición gobernante a la luz de la literatura en Ciencia Política sobre coaliciones (sección 2). Dada la diversidad de preferencias sobre políticas que conviven al interior de un gobierno de coalición, la literatura esperaría que los socios utilicen debates legislativos y otras oportunidades para vigilar a sus socios y comunicarse con su electorado.

Segundo, analizamos los sucesos principales que tuvieron lugar en relación a tres grandes desafíos que enfrentó el nuevo gobierno durante el año 2020 y parte del 2021: el manejo de la pandemia por COVID-19 (sección 3), la renegociación de la deuda pública en manos extranjeras (sección 4) y la relación con una región que había virado considerablemente hacia a la derecha ideológica, al menos en relación a la última vez que el peronismo estuvo en el poder (sección 5). Si bien no es nuestro objetivo utilizar estas tres áreas como casos para testear argumentos teóricos sobre las causas y efectos de las coaliciones, encontramos evidencia que se acomoda a las expectativas de la literatura sobre la conducta de los miembros de un gobierno coalicional, especialmente cuando las políticas en cuestión tocan temas sobre los cuales los miembros mantienen fuertes preferencias establecidas. Por ejemplo, se observa que las instancias en el manejo de la pandemia o la renegociación de la deuda externa en las que las políticas sociales tomaron prominencia coincidieron con mayores cuestionamientos por parte de figuras y grupos de la coalición alineados con el kirchnerismo. En el mismo sentido, pronunciamientos sobre el régimen venezolano generaron reclamos similares en relación a la política exterior regional.

\section{EL FRENTE DE TODOS SEGÚN LA LITERATURA SOBRE COALICIONES}

La literatura sobre coaliciones ofrece herramientas teóricas para entender tanto el origen de una coalición como los efectos que podría tener sobre el proceso de producción de políticas y sobre la gobernabilidad en sentido más amplio (Laver y Shepsle 1990; Cheibub, Przeworski, y Saiegh 2004; Martin y Vanberg 2004; Negretto 2006). Una coalición se forma cuando dos o más actores deciden coordinar su accionar bajo el presupuesto que actuar de manera concertada les permitirá alcanzar un objetivo que no podrían alcanzar individualmente. Las características institucionales del sistema político pueden incentivar a los actores a formar coaliciones de distinto tipo y en diferentes momentos. Por ejemplo, en un sistema presidencialista, partidos que consideren que su potencial de votos no será suficiente para ganar la elección, se verán incentivados a formar 
coaliciones pre-electorales con otros candidatos y partidos y así compartir recursos y coordinar estrategias de campaña (Borges, Turgeon, y Albala 2020). ${ }^{1}$

En las últimas décadas, la política argentina y en particular las estrategias electorales han estado marcadas por dinámicas de tipo coalicional (Clerici 2015; Cruz 2019). El origen de la actual coalición gobernante, de modo análogo a las experiencias anteriores, se encuentra en muchos de los incentivos previstos en la literatura. ${ }^{2}$ La experiencia de las elecciones presidenciales del 2015 y legislativas del 2019 reveló al Frente para la Victoria (FpV) de CF que volver al poder solo sería posible a través de una coalición más amplia. En 2015 el peronismo se había divido, con Daniel Scioli representando al FpV y Sergio Massa a los peronistas federales y disidentes reunidos en el Frente Renovador (FR). Scioli obtuvo $37.1 \%$ de los votos pero ello no fue suficiente para ganar la presidencia en primera vuelta y tampoco fue capaz de atraer la cantidad necesaria de votantes del FR y otros partidos en la segunda. Dos años más tarde, en la elección legislativa de 2017, la dinámica fue similar. Si bien CF hizo un llamado a la construcción de una nueva coalición amplia para oponerse al gobierno de Cambiemos, finalmente no logró incorporar a los peronismos disidentes encabezados por el FR, que junto a otros partidos menores como Generación para un Encuentro Nacional (GEN) o Libres del Sur, se presentaron como la coalición "1País." La derrota en la Provincia de Buenos Aires indicaría otra vez las limitaciones del peronismo dividido. Finalmente, el regreso a la presidencia en las elecciones de 2019 sucedió a partir de la conformación del Frente de Todos (FdT), incluyendo tanto a las fuerzas kirchneristas alrededor del FpV pero también al FR, sectores del peronismo federal y a otros socios menores, tanto del campo peronista como fuera de él (Anria y Vommaro 2020).

Para poder alcanzar esta unidad el rol de CF fue decisivo, particularmente como consecuencia de su decisión de ceder el centro de la escena y ofrecer a AF la posibilidad de encabezar la fórmula presidencial. En conformidad con la tendencia a la personalización de la oferta electoral que nota Gervasoni (2018), la percepción de AF como un líder moderado, que había sido tanto Jefe de Gabinete durante los gobiernos kirchneristas así como también jefe de campaña de Sergio Massa en 2015, otorgaba un perfil más centrista que permitía la reincorporación de los diferentes sectores peronistas, en particular la del FR. Conformar una coalición es un proceso transaccional en el que las partes acuerdan gobernar conjuntamente si el pacto electoral resulta exitoso. Pero gobernar en conjunto tiene costos: todos menos uno de los partidos que confor-

Estos incentivos contrastan con los que se provocan en sistemas parlamentarios donde las coaliciones tienden a formarse después de las elecciones.

Es necesario hacer una aclaración en relación a la aplicabilidad de los argumentos teóricos sobre coaliciones al actual sistema político argentino. Si bien los partidos políticos son los actores principales en la literatura, el sistema de partidos argentino ha atravesado un fuerte proceso de desinstitucionalización en las últimas décadas. Como resultado, puede ser más apropiado considerar como actores a líderes individuales y facciones que se mueven de un partido a otro o crean nuevos partidos con relativa fluidez (Gervasoni 2018). Hecha esa aclaración, asumimos que los argumentos acerca de cuántos deberían unirse para competir, con quién y con qué efectos también deberían aplicar a líderes y grupos más laxos. 
man la coalición pierden la posibilidad de presentar un candidato a presidente (Albala 2021). Incluso el partido que obtiene la candidatura presidencial debe renunciar a ocupar algunas de las posiciones en un eventual gabinete o debe prometer ciertas políticas que los socios valoran y que tal vez no hubiesen sido prioritarias en un gobierno de partido único (Kellam 2017). Dados estos costos, la literatura sugiere que a la hora de formar una coalición los partidos van a considerar dos factores principales con tal de reducir la distancia entre su distribución de posiciones de gabinete y políticas ideal -teóricamente, aquella que tendría lugar si gobernaran solos- y la distribución esperada de gobernar en coalición. Primero, las coaliciones van a tender a incluir el menor número posible de socios necesarios para alcanzar el objetivo electoral (Riker 1962). Segundo, es más probable que los actores que terminen formando una coalición sean aquellos con menor distancia relativa entre sus preferencias (Axelrod 1970).

El acuerdo entre el FpV, el FR y otros socios menores de unirse detrás de la candidatura de AF ilustra estas dinámicas coalicionales pero también señala los límites de argumentos que asumen que los actores cuentan con la información necesaria para estimar la fuerza relativa de potenciales socios. En el caso argentino, el faccionalismo, personalismo y la creciente fluidez del sistema de partidos debería dificultar estos cálculos (Gervasoni 2018). En cuanto a las preferencias, que la mayoría de los participantes se identifiquen con el peronismo sugiere que las diferencias entre ellos son menores en comparación a las que existen con otros partidos y candidatos que adoptan una identidad en oposición al peronismo. De todas formas, la identidad peronista compartida no elimina las diferencias hacia el interior de la coalición. Si bien el peronismo históricamente ha mantenido una estructura ideológica flexible que facilita la convivencia de diferentes preferencias en su interior, que el peronismo se hubiera presentado dividido en prácticamente la totalidad de las elecciones posteriores al 2001 indica que las divergencias pueden llegar a generar costosas rupturas.

La inclusión en una misma coalición de sectores con intereses muchas veces contrapuestos tiende a generar tensiones durante el ejercicio del gobierno. En este caso, diferencias sobre políticas podrían surgir entre representantes de los trabajadores formales afiliados a sindicatos, muchos de los cuales habían apoyado al FR cuando el peronismo estuvo dividido ${ }^{3}$ y los representantes de los movimientos sociales alineados con el FpV (Zarazaga 2019; Murillo y Zarazaga 2020). Asimismo, este tipo de conflictos tiende a agudizarse cuando la economía se contrae. Argentina ya atravesaba un periodo de mal desempeño económico cuando el nuevo gobierno llegó al poder y se sumaría a esta situación el impacto económico de la pandemia global.

Respecto del clivaje formal-informal, si bien Etchemendy argumenta que el kirchnerismo fue capaz en cierta medida de contrarrestar la fragmentación del sector popular manteniendo tanto a sindicatos como movimientos sociales en su coalición, también reconoce que el kirchnerismo "nunca institucionalizó su relación con los sindicatos y movimientos sociales" (2020: 29). 
La heterogeneidad al interior del Frente de Todos aumenta aún más en la medida que también se incorporaron trece gobernadores provinciales, incluyendo a aquellos que habían comenzado a negociar una alternativa al peronismo kirchnerista pero finalmente desistieron de buscar vías alternativas. Los puntos de tensión no giraban solo en torno a políticas económicas y sociales, sino que también se vislumbraban diferencias en otras áreas donde entran en juego diferencias sobre valores o clivajes de corte netamente ideológico. En este sentido, la legalización de la interrupción voluntaria del embarazo había sido una promesa de campaña de AF que se preveía generaría posiciones encontradas dentro de la coalición. ${ }^{4}$ Aun cuando la ley fue aprobada teniendo por principal apoyo a los legisladores del Frente de Todos, las divisiones internas dentro de la coalición fueron claras. Si bien el apoyo explícito del presidente y la vicepresidenta al proyecto de ley marcaban la posición mayoritaria, la oposición de figuras prominentes como el jefe de bloque en Senadores, José Mayans, el entonces presidente del Partido Justicialista, José Luis Gioja, y otros gobernadores, mostraban esta diferencia de criterios.

Otro rasgo distintivo de las coaliciones de gobierno son los problemas de credibilidad y delegación. Como se mencionó, formar una coalición implica una transacción. Las transacciones que subyacen a coaliciones pre-electorales presidencialistas sufren particularmente de problemas de credibilidad ya que a diferencia de lo que sucede en los parlamentarismos, los partidos no tienen mecanismos institucionalizados para castigar al presidente si no cumple con el acuerdo pre-electoral (Kellam 2017). El riesgo que acarrea la delegación de poder a otros miembros de la coalición genera incentivos para que cada socio vigile las acciones de funcionarios pertenecientes a otros sectores. De acuerdo a Martin y Vanberg $(2004 ; 2008)$, el proceso legislativo permite a los miembros de la coalición vigilar lo que hacen los funcionarios pertenecientes a otros sectores y provee oportunidades para comunicarse con su electorado y justificar compromisos desfavorables. De hecho, los debates legislativos sobre la ley para la interrupción voluntaria del embarazo permitieron a la minoría de miembros de la coalición en contra de la aprobación de la ley comunicar su desacuerdo con la posición mayoritaria. En este punto, es particularmente claro el caso de legisladores dentro de la propia coalición oficialista que perteneciendo a provincias con electorados predominantemente opuestos a la ley, utilizaron el espacio para marcar su diferencia.

En el caso del actual gobierno, se da una particularidad que puede afectar la severidad de los problemas de delegación. En coaliciones pre-electorales presidenciales, la expectativa es que la candidatura a presidente -siendo la posición con mayor autoridad- la ocupe el socio mayoritario. Sin embargo, AF no proviene del partido mayoritario, el FpV, ni tiene fuerza territorial propia. Esto ge- 
nera que si bien el poder de decisión final reside con el presidente, CF mantiene poder de veto sobre decisiones políticas particulares como líder de la primera minoría electoral. Aunque esta distribución de poderes formales y de facto podría solucionar el problema de delegación que este tipo de arreglo coalicional significaría para el kirchnerismo, también puede intensificar los desacuerdos sobre políticas ya que no está predeterminado que la posición de quien ocupa la presidencia es la que va a terminar imponiéndose.

En resumen, de lo examinado en esta sección se desprenden dos atributos principales del actual gobierno que, sugiere la literatura, podrían afectar el funcionamiento de la coalición y la toma de decisiones. Primero, la multiplicidad de partidos, grupos y personalidades que forma parte de esta coalición resulta en la coexistencia de una multiplicidad de preferencias que, en algunos casos, pueden ser divergentes. Segundo, y en combinación con lo anterior, la distribución de autoridad formal -cargos y control sobre recursos- e informal -apoyo popular- entre actores con preferencias diversas genera incentivos para que los socios se vigilen unos a otros, comuniquen sus desacuerdos y justifiquen a sus votantes cuando el gobierno del que participan toma decisiones con las que no concuerdan. A continuación, examinamos los principales acontecimientos en el manejo de la pandemia, la renegociación de la deuda y la política exterior regional prestando especial atención a la manifestación de estas dinámicas típicas de gobiernos de coalición como el actual.

\section{EL MANEJO DE LA PANDEMIA: CUARENTENA Y VACUNACIÓN}

La pandemia por COVID-19 fue el principal hecho que marcó la vida social, política y económica en el año 2020 en todo el mundo. Los gobiernos debieron ensayar respuestas a un fenómeno inédito que afectaba a todos los aspectos de la vida diaria doméstica, así como también sus relaciones externas. Al momento de la asunción presidencial, el gobierno argentino enfrentaba además una delicada situación económica y social. La actividad económica se había contraído durante dos años seguidos, la inflación no cedía luego de más de diez años con figuras por encima de los dos dígitos, y si bien el crónico déficit fiscal había disminuido, la contracara había sido el aumento de la deuda pública en moneda extranjera, que se acercaba al 70\% del PBI a fines de 2019 (Secretaría de Finanzas 2019; INDEC 2020a). La pobreza, por su parte, se mostraba en aumento luego del descenso de 2016 y 2017, alcanzando a más del 25\% de los hogares y más del 35\% de las personas al momento de la asunción del nuevo gobierno (INDEC 2020c).

En un contexto económico y social ya deteriorado, los primeros casos de COVID-19 en Argentina se registraron durante los primeros días de marzo de 2020. Frente a esto, el gobierno dispuso el 15 de marzo el cierre de fronteras para no nacionales, la suspensión de educación presencial a nivel nacional y la cancelación de actividades no esenciales y aglomeraciones hasta fin del mismo 
mes. Esto fue acompañado además por medidas dirigidas a contener el daño económico que generaría la implementación del Aislamiento Social Preventivo y Obligatorio (ASPO) en todo el territorio, tal como se verá más adelante. Esta primera etapa se encuentra marcada por una fuerte aceptación de la cuarentena por parte de la opinión pública aún a pesar del fuerte impacto económico generado por las medidas (Schraff 2020). Probablemente ayudó a generar este resultado que en este periodo la oposición política disminuyó sus críticas al gobierno y las medidas sanitarias mostraron altos niveles de coordinación con los gobiernos opositores subnacionales.

Figura 1: Evolución de la Actividad Económica y Confianza en el Gobierno

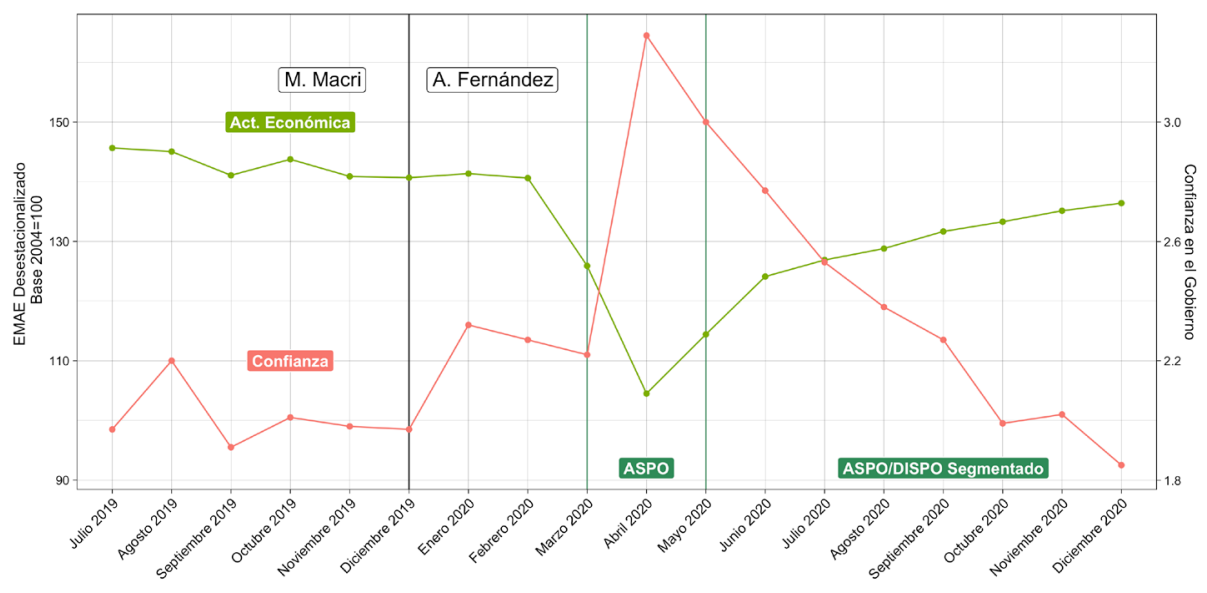

La rápida respuesta del poder ejecutivo frente a la pandemia generó una buena recepción en la población, en tanto la aprobación del presidente y la confianza en el gobierno alcanzaron sus picos máximos en marzo de 2020, marcando un notable crecimiento con respecto a meses anteriores. Sin embargo, los datos también muestran que ese pico de popularidad fue efímero. El Índice de Confianza en el Gobierno, elaborado por la Universidad Torcuato Di Tella (2021), creció en más de 48 puntos porcentuales entre marzo y abril, pero desde entonces mostró un deterioro sostenido hasta fin de año (ver Figura 1). La Encuesta de Satisfacción Política y Opinión Pública de la Universidad de San Andrés (2020a) muestra números similares: la satisfacción sobre la marcha general de las cosas creció hasta alcanzar un pico de $57 \%$ en abril para luego caer a $21 \%$ en octubre y mantenerse en valores similares hasta fin de año. La aprobación del gobierno, por su parte, pasó de $67 \%$ a $32 \%$ en el mismo período, alcanzando el punto más bajo de todo su gobierno, incluso a niveles similares a los de Mauricio Macri en el piso de su popularidad en 2019.

Las razones de la abrupta caída parecen estar relacionadas con una efímera atención en el COVID-19 y por la vuelta al primer plano de las preocupaciones 
económicas y de seguridad: mientras en abril la dimensión sanitaria de la pandemia aparecía al tope de las preocupaciones, en julio los principales problemas que planteaban los encuestados volvían a ser la corrupción, la inseguridad, el desempleo y la inflación, agravadas estas últimas por el impacto negativo de las medidas ASPO sobre estas áreas. Por su parte, las estadísticas sobre la situación social avalaban esta preocupación en tanto la pobreza alcanzó al 40.9\% y la indigencia al 10.5\% de la población en el primer semestre de 2020, mientras que el desempleo se mantuvo por arriba del 10\% durante los primeros nueve meses del 2020, aunque presentó un leve descenso entre el segundo y tercer trimestre (INDEC 2020b, 2020c).

Frente a las consecuencias sobre la situación económica de las restricciones a la movilidad y al cierre de diversas actividades, el gobierno buscó implementar una serie de medidas de mitigación, tales como duplicar el monto de la Asignación Universal por Hijo o decretar la suspensión de los cortes de servicios por 180 días, y la implementación de planes específicos para la situación de la pandemia, como el Programa de Asistencia de Emergencia al Trabajo y la Producción (ATP), orientado a la asistencia en el pago de salarios y el otorgamiento de créditos, o el Ingreso Federal de Emergencia (IFE), destinado a desempleados, trabajadores informales y cuentapropistas de bajos ingresos -una parte sustancial del electorado del FpV- con el objeto de contener las causas negativas de las restricciones entre los sectores más vulnerables. El IFE se pagó en tres oportunidades, en abril, junio y agosto, teniendo un monto fijo equivalente a poco más de dos veces la Canasta Básica Alimentaria del momento. En la primera aplicación del IFE se efectivizaron casi 8.9 millones de asignaciones, lo que muestra una amplia cobertura de la medida (ANSES 2020).

No obstante, la decisión de no otorgar el IFE por una cuarta vez originó críticas desde la propia coalición, específicamente en los sectores más identificados con los trabajadores de la economía informal, que resaltaron el aumento de la pobreza y la indigencia y acusaron al gobierno de "ver la realidad con el Excel y mirando más a la señora del Fondo Monetario Internacional que a la señora del barrio" (Infobae 2020c; Perfil 2020a). La explicación del ministro de Economía, Martín Guzmán, expresando preocupaciones por la disciplina fiscal en medio de negociaciones por la deuda externa, había referido a que no se implementaba un cuarto IFE porque era necesario "mantener ciertos equilibrios", aunque no descartaba volver a implementarlo ante una nueva ola de la pandemia (Cronista 2020). Meses después, en medio de la tercera ola, la agrupación kirchnerista La Cámpora reiteró el pedido por una nueva ronda del IFE (Infobae 2021). Cuestionamientos de este tipo por parte del ala kirchnerista de la coalición ocurrieron con cierta regularidad durante el primer año de la pandemia. De acuerdo a las expectativas de Martin y Vanberg (2004), los debates en el Congreso sirvieron para que miembros de la coalición vigilen funcionarios que no controlan y les traspasen su desacuerdo con decisiones particulares. Por ejemplo, durante sesiones en la Cámara de Diputados, Máximo Kirchner apro- 
vechó la oportunidad para cuestionar al ministro de Trabajo, Claudio Moroni, por haber aprobado un acuerdo entre una empresa y sus empleados que incluía una reducción salarial en un contexto de crisis (La Política Online 2020).

Otra de las medidas que estuvieron en debate para contrarrestar los efectos negativos de las restricciones durante la segunda mitad del año fue la implementación de un Aporte Solidario y Extraordinario, coloquialmente denominado "impuesto a las grandes fortunas," a partir de un proyecto surgido del kirchnerismo. Esta propuesta generó un fuerte rechazo en la oposición, pero también de diferentes sectores productivos, incluyendo organizaciones de productores agropecuarios, como la Sociedad Rural, la Unión Industrial, la Asociación Empresaria, y las Cámaras de la Construcción y el Comercio (Infobae 2020e). Finalmente la ley fue aprobada a principios de diciembre, aunque con el rechazo de prácticamente la totalidad del bloque opositor.

En línea con esto último, cabe señalar que las críticas más fuertes provinieron de la oposición, incluso en aquellos temas que generaban divisiones internas de la coalición oficialista. En este marco, se dieron una serie de protestas en contra de las medidas del gobierno para controlar la pandemia, que si bien no alcanzaron niveles masivos, tuvieron una gran repercusión mediática y apoyo de algunos sectores de la oposición. En estos últimos, la presidenta del PRO Patricia Bullrich fue una de las principales voces en apuntar al accionar del gobierno tanto en relación a la implementación del ASPO como en la gestión de las vacunas para hacer frente a la pandemia. Dentro de estas críticas, una de las más recurrentes y duras fue la relacionada con la decisión del gobierno nacional de mantener las escuelas cerradas en el área metropolitana de Buenos Aires. Si bien en un principio la suspensión de las clases tuvo una buena recepción general, se observaban claras diferencias entre los sectores oficialistas y opositores, donde estos últimos planteaban menor apoyo a la medida (Universidad de San Andrés 2020b). Durante el segundo semestre, la falta de flexibilización sobre los cierres y la ausencia de propuestas de semipresencialidad o vuelta presencial a las escuelas comenzó a ser foco creciente de críticas e incluso el apoyo a la medida dentro también de las bases oficialistas comenzó a flaquear. En una encuesta realizada en marzo de 2021 solo un $29 \%$ de los votantes de AF apoyaban la modalidad completamente virtual, mientras que un $25 \%$ y un $39 \%$ se decantaban por la presencialidad con distancia social y un sistema semi-presencial, respectivamente (Universidad de San Andrés 2021).

Así como durante los primeros meses de la pandemia las principales críticas al gobierno se centraban en el mantenimiento de medidas rígidas, especialmente en el área metropolitana de Buenos Aires, y el impacto que esto tenía sobre la economía y la situación social, en el segundo semestre el debate viró hacia el acceso a las vacunas. En este tema, durante el año 2020 Argentina pudo avanzar sus gestiones en relación a dos vacunas diferentes. En el mes de agosto, el presidente anunció que la vacuna que estaba siendo desarrollada por la Universidad de Oxford y AstraZeneca sería fabricada en conjunto 
por Argentina, donde se produciría el principio activo, y México, donde se envasaría y distribuiría. Esta primera vacuna implicó sin embargo tiempos más lentos al de otras opciones, en tanto recién se aprobó para su uso de emergencia en los últimos días de diciembre y los envíos de los primeros lotes de principios reactivos fueron realizados en los primeros meses de 2021. En paralelo, en los últimos días de febrero llegaron 580 mil dosis de la vacuna Covishield desde la India, también elaborada a partir de la tecnología del desarrollo de Oxford y AstraZeneca.

La segunda vía de acceso a vacunas fueron las gestiones para adquirir la Sputnik V, desarrollada por el instituto ruso Gamaleya. Esta se transformaría en la primera vacuna que efectivamente llegaría y se aplicaría en el país. En los primeros días de noviembre el gobierno anunció que estaba en negociaciones para comprar 25 millones de dosis de esta vacuna. Un mes más tarde, Argentina finalmente firmaba el acuerdo con el Fondo Soberano de la Federación Rusa por un total de 10 millones de vacunas a ser entregadas en enero y febrero de 2021, con la opción de 5 millones más de vacunas para el mes de marzo. En esta ocasión, Fernández agradeció expresamente a Vladimir Putin, quien "se ocupó personalmente de ayudar a que esto ocurra," al tiempo que resaltaba que esto le permitía al país "acceder a la vacuna al mismo tiempo que el mundo central" (MRECIC 2020). En este punto, cabe resaltar que la adquisición de la Sputnik V generó fuertes críticas en sectores de la oposición, que señalaban que los resultados de su aplicación eran insuficientes y poco transparentes, lo que también se exacerbó a partir de la autorización tardía para utilizarla en mayores de 60 años (El País 2020b; La Nación 2020a).

El manejo de la pandemia se encontró entonces estructurado en tres etapas. En la primera, la urgencia sanitaria y la incertidumbre favorecieron la toma de decisiones por parte del presidente ya que redujeron la posibilidad de conflicto, tanto desde dentro como fuera de la coalición. En la segunda, la reaparición de las preocupaciones económicas generó cuestionamientos desde la oposición pero también desde el interior de la coalición, particularmente desde el kirchnerismo y los representantes de los movimientos sociales que reclamaron la expansión de la ayuda estatal a los sectores de menores ingresos. En la tercera etapa, la apuesta por la vacuna Sputnik V generó grandes críticas desde la oposición dada la falta de información y la desconfianza hacia el régimen ruso. Finalmente, la publicación de los resultados de los ensayos clínicos en The Lancet en los primeros días de febrero de 2021 terminaron por disipar las dudas sobre su efecto y seguridad. Más allá de esto, las vacunas continuarían siendo el centro de la puja política durante el 2021 en dos aspectos: el número en que llegarían y como serían distribuidas. Aquí la principal disputa aparecería nuevamente entre jurisdicciones, y especialmente entre la Ciudad de Buenos Aires, en manos de la oposición, y el gobierno nacional. 


\section{DOBLE DEUDA: ACREEDORES PRIVADOS EXTRANJEROS Y EL FONDO MONETARIO INTERNACIONAL}

En las primeras semanas del gobierno de AF ya había quedado claro que resolver la cuestión de la deuda en manos de acreedores extranjeros sería una prioridad. Un indicador de la importancia de este tema es que ya en enero de 2020 el presidente viajó a varios países europeos y en todas las reuniones que mantuvo mencionó el problema de la deuda y se aseguró de obtener una declaración de apoyo de sus contrapartes. ${ }^{5}$ La renegociación de la deuda estuvo concentrada en manos de los funcionarios de más alto nivel: el ministro de Economía, Martín Guzmán, y el propio presidente, provocando una alta concentración de la información sobre cómo evolucionaban los términos de las negociaciones.

Argentina tenía dos frentes principales en este ámbito. El primero, y más urgente ya que tenía un primer vencimiento en el mes de mayo, era la deuda con los acreedores privados. El segundo era la deuda con el FMI que recién tendría un primer vencimiento en 2021. El punto de partida del gobierno era que ambas deudas eran insostenibles y, dado este diagnóstico, la única opción era una renegociación. Este proceso, como una gran parte de las interacciones políticas, puede entenderse como implicando al mismo tiempo interacciones cooperativas y de regateo. ${ }^{6}$ Todas las partes se beneficiarían de un acuerdo ya que el status quo avecinaba un default. Al mismo tiempo, toda negociación de deuda incluye un fuerte componente de regateo en el que las ganancias de una parte resultan en pérdidas para la otra. Los acreedores, por ejemplo, preferían períodos de gracia más cortos -entendidos como el tiempo durante el cual el estado no haría nuevos pagos- y quitas a los intereses lo más bajas posibles; mientras que el interés del gobierno va en la dirección opuesta. Además de la complejidad intrínseca de este tipo de negociaciones, se suma el hecho que, en el caso de la deuda con privados, los acreedores están atomizados en un gran número de tenedores de bonos (Panizza, Sturzenegger, y Zettelmeyer 2009). Adicionalmente, en toda interacción de regateo, es crucial la cuestión de la credibilidad de las posiciones de las partes cuando indican sus límites de tolerancia.

La estrategia argentina fue vincular ambas deudas lo más posible. El gobierno buscó que el Fondo avale su diagnóstico sobre la incapacidad de Argentina de hacer frente a sus obligaciones. El gobierno enfrentaba un problema de credibilidad en este aspecto ya que tenía incentivos para presentar su situación económica en la peor luz posible. Cuánto menos se esperaba que crezca Argentina en el futuro, más grande debería ser la quita en la negociación actual. Se esperaba que si el Fondo llegase a esta misma conclusión, la posición Argentina ganaría credibilidad frente a los acreedores. Esto terminó ocurriendo en febrero tras una visita de un equipo técnico del FMI que determinó

Alberto Fernández discutió la cuestión de la deuda en sus encuentros en el Vaticano, Italia, España, Alemania y Francia. Además, el nuevo Embajador frente a los Estados Unidos, Jorge Argüello, también se aseguró de mencionar el tema en su primer encuentro con Donald Trump. Ver (El País 2020a; Perfil 2020b).

Sobre cooperación y regateo simultáneo, ver Fearon (1998). 
que el superávit fiscal necesario para hacer frente a estas obligaciones no era factible en términos económicos ni políticos. Se reconoció también que resolver el problema de la deuda requeriría una "contribución apreciable de los acreedores privados". Este pronunciamiento podría parecer inesperado ya que implica el respaldo a un gobierno que se apoya mayoritariamente en sectores críticos del FMI. De hecho, existieron pronunciamientos de ese tenor por parte de Cristina y Máximo Kirchner al tiempo que distintas agrupaciones sociales protestaron la visita de los técnicos del Fondo (La Nación 2020b). De todas formas, durante esta primera renegociación el extremo de la coalición más resistente a una relación cercana con el FMI se mantuvo relativamente aislado de la cuestión de la deuda dando al presidente y al ministro amplio margen para negociar. Si bien es difícil explicar en este momento por qué el Fondo decidió tomar esa posición, es necesario considerar que el gobierno había dado señales al FMI que existía la disposición para reducir en cierta medida el déficit fiscal. Por ejemplo, entre las primeras medidas económicas del gobierno estuvieron suspender la cláusula de ajuste de las jubilaciones, subir impuestos y retenciones a las exportaciones y excluir a los empleados públicos del régimen de doble indemnización por despidos.

Además del respaldo del FMI, la crisis económica generada por la pandemia global contribuía al diagnóstico argentino de que la deuda era insostenible. Tanto el presidente como el ministro Guzmán resaltaron repetidamente los efectos económicos de la pandemia. ${ }^{7}$ El énfasis en los factores exógenos sugiere un intento por apelar a la tendencia de los inversores a castigar menos severamente a deudores que cesan sus pagos sin justificaciones económicas válidas (Tomz 2007). En este contexto, en abril comenzaron las negociaciones. La primera oferta por parte del estado implicaba un recorte del $62 \%$ a los pagos por intereses, 5.4\% reducción en el valor de la deuda y un periodo de gracia de 3 años. En conjunto, Argentina ofrecía alrededor de 40 dólares por cada 100.

En los meses siguientes continuaron las ofertas y contra-ofertas de las partes; un proceso además complejizado por las diferentes posiciones tomadas por distintos grupos de acreedores. Cada vez que el ministro Guzmán realizaba una nueva oferta -48 dólares cada 100, luego 53 y finalmente 54.8- reiteraba la advertencia que era imposible mejorar los términos y que esta era la última oferta. En la negativa de los bonistas a las sucesivas ofertas se evidenciaba la creencia que Argentina podía hacer mayores concesiones, aunque los acreedores también moderaron sus demandas en las sucesivas contra-ofertas, bajando en un principio a 55 dólares por cada 100, pero manteniendo también la posibilidad de acciones legales sobre la mesa en todo momento. Durante este periodo la distancia entre las partes se fue achicando mientras que el FMI seguía apoyando la posición de Argentina, declarando luego de la primera oferta del gobierno que quedaba "un margen limitado para incrementar los pagos a los acreedores privados" (FMI 2020). El acuerdo final se alcanzó en agosto cuando 
Argentina propuso pagar 54.8 dólares cada 100 y los acreedores comenzaron uno tras otro a aceptar la oferta.

Es imposible saber si Argentina realmente consideraba su oferta inicial como viable o si se trataba meramente de un punto de partida artificialmente ambicioso para tener luego espacio para hacer concesiones. De todas formas, dada la información existente acerca de las trayectorias de las partes durante la negociación, se observa que fue el estado quien recorrió mayor distancia desde su posición inicial. En este sentido, el respaldo del FMI durante el proceso no parece haber sido suficiente como para que el acuerdo final se hubiese ubicado más lejos de la posición inicial de los acreedores que de la de Argentina. De todas formas, en ausencia de un contrafáctico es imposible saber si los acreedores hubiesen sido más duros aún en su ausencia. Otro dato interesante sobre el comportamiento del Fondo en este tema es que, si bien se trata del organismo internacional con peor reputación en Argentina, sondeos de opinión pública sugieren que los valores positivos subieron entre mediciones de octubre 2019 y abril 2020 (Poliarquía \& Wilson Center 2020).

Una vez cerrado el frente con los acreedores privados, la negociación con el FMI se convirtió en la prioridad. Por varias razones, esta negociación probaría ser un proceso más complejo. No sólo se trata de un volumen de deuda mayor, sino que el FMI es particularmente impopular dentro del kirchnerismo donde la narrativa dominante es que el Fondo es responsable de muchos de los problemas económicos de Argentina. En contraste con esta posición, durante la campaña electoral AF había enviado señales de moderación y pragmatismo al FMI anunciando que su única motivación sería alcanzar un acuerdo que funcione para todas las partes. Más allá de este discurso más moderado, hacia fines del 2019 la opinión pública sobre el FMI exhibía altos valores de polarización, yendo de $72 \%$ de imagen positiva entre quienes aprobaban la figura del presidente saliente Mauricio Macri a 14\% entre quienes lo desaprobaban (Poliarquía \& Wilson Center 2019). En el gobierno entendían que la llegada al poder en 2019 se debió, en gran parte, a que una porción del electorado castigó al gobierno de Macri por la mala situación económica (Garriga y Negri 2020). Con miras a las elecciones legislativas de finales de 2021, moderar la inflación aparecía como una meta necesaria para evitar un castigo similar. Al mismo tiempo, las tensiones inflacionarias, entre otras razones, responden a que mientras el país no pueda acceder al crédito externo el gasto público -lógicamente incrementado durante la pandemia- debe financiarse a través de la emisión. De ahí que llegar a un acuerdo con el Fondo que permita a Argentina volver a colocar deuda en el exterior es funcional electoralmente. El dilema que esto genera para el gobierno es que los términos de un acuerdo con el Fondo provocan tensiones al interior de la coalición más que nada en relación a cómo generar el superávit fiscal necesario para afrontar los pagos. Elegir estos instrumentos es fundamentalmente un problema distributivo. Las medidas disponibles -impuestos a las clases más altas o a las actividades extractivas de las provincias, retenciones a las exportaciones agrarias, reducción de programas sociales y jubilaciones o 
disminución del ingreso de empleados públicos- concentran los costos en diferentes grupos sociales (Biglaiser y McGauvran 2021).

La relación con el FMI, concentrada fuertemente en manos de un ministro de Economía que no proviene de los sectores más críticos al organismo, dio lugar a diferentes pronunciamientos durante la primera mitad del 2021 que reflejan la tendencia de los miembros de la coalición a comunicar con políticas desfavorables. Por ejemplo, el senador kirchnerista Oscar Parrili impulsó en el Senado la adopción de una declaración que solicita al Ministerio de Economía utilizar fondos del FMI para políticas sanitarias y sociales y no para el pago de la deuda (Cronista 2021b). Los reclamos desde el interior de la propia coalición se repitieron en varias instancias a la sombra de las negociaciones con el FMI. ${ }^{8}$

\section{LA INSERCIÓN REGIONAL: AMÉRICA LATINA ENTRE LA INESTABILIDAD Y EL GIRO A LA DERECHA}

En adición a las limitaciones que imponían la pandemia, la delicada situación económica y social, y la necesidad imperiosa de renegociar la deuda, el gobierno de AF enfrentaba tres limitaciones principales en su inserción regional. En primer lugar, la vuelta del peronismo se encontró rodeada por gobiernos que, aun con diferencias entre sí, representaban en cada uno de sus países a partidos y electorados ubicados a la derecha del centro ideológico: Piñera en Chile, Añez en Bolivia, Abdo en Paraguay, Bolsonaro en Brasil y Lacalle Pou en Uruguay. Si tomamos a toda la región, y dejando de lado el caso de Venezuela tanto por espinoso en cuestiones domésticas como por su poca capacidad de acción externa, tan solo López Obrador en México parecía ofrecer un interlocutor con una visión similar del mundo al gobierno argentino. Este aislamiento se evidenció rápidamente, incluso antes de asumir la presidencia, cuando AF visitó en noviembre de 2019 a su par mexicano y marcaron públicamente sus coincidencias y la necesidad de fortalecer la relación bilateral. En segundo lugar, la región presentaba fuertes signos de inestabilidad en las instituciones y competencia política democráticas. Al ya largo retroceso y finalmente reversión de la democracia venezolana, se sumaron en enero de 2019 la llegada de Bolsonaro al poder en Brasil con un discurso reivindicativo de las dictaduras militares y opuesto en muchos aspectos a los derechos humanos, y en noviembre las turbulentas elecciones presidenciales en Bolivia y el eventual golpe de estado contra Evo Morales que permitió a Añez acceder al poder. Por su parte, los fuertes estallidos sociales en Ecuador, Perú y, especialmente, Chile, fueron respondidos por los gobiernos con una represión que fue denunciada por organismos internacionales de derechos humanos (CIDH 2019a; 2019b; 2020). Además de la amenaza que representa que la inestabilidad social se expanda por la región, los estallidos sociales y retrocesos democráticos en la región le planteaban al gobierno el problema de cómo posicionarse en cada uno de estos 
casos, manteniendo un delicado balance entre las limitaciones que le imponen los diferentes sectores de su coalición doméstica y los costos que a nivel externo puede generar en las relaciones bilaterales.

En tercer lugar, el COVID-19 terminó de coronar una situación regional ya de por sí desafiante, al agregar dos temas nuevos en la agenda: la contención de los efectos negativos de la pandemia y el posterior acceso a las vacunas y los tratamientos. Esto aparecía como un arma de doble filo en tanto planteaba un nuevo punto en donde confrontar con los gobiernos vecinos, al tiempo que podía ofrecer un área en la que cooperar más allá de las diferencias ideológicas, dada la magnitud del problema.

Como producto de la conformación de un frente amplio para alcanzar el poder, el gobierno de AF presentaba, al menos, dos visiones diferentes sobre la política exterior (Merke 2019). La primera, menos claramente formulada en un principio, estaba representada por el mismo presidente y su canciller, Felipe Solá, quien provenía de las filas del FR luego de también haberse alejado del kirchnerismo. La segunda era más discernible ya que implicaba una continuidad con la política exterior del gobierno de Cristina. ${ }^{9}$ Como en otras cuestiones, las preferencias del grupo alrededor del presidente se inclinarían por moderar algunos de los rasgos salientes de una política exterior de los gobiernos de CF. Algunas de estas diferencias se pudieron observar ya durante la campaña presidencial en diversos ámbitos. En primer lugar, AF buscó entablar una relación más cooperativa con las instituciones financieras internacionales y encarar una inevitable renegociación con los acreedores privados con un tono más conciliador que lo que se observó durante la última presidencia de $\mathrm{CF}$, tal como se presentó en la sección previa. En cuanto a la relación con los grandes centros de poder, el futuro Embajador argentino en Washington, Jorge Argüello (2019), señalaba la necesidad de una posición equilibrada y de "medir con cuidado la tensión chino-estadounidense, eludir la tentación de apoyarse en solo una de las dos potencias". Sobre el acuerdo comercial entre el Mercosur y la Unión Europea firmado a mediados de 2019, AF se mostró favorable a avanzar sobre el mismo tanto en la campaña como durante varias ocasiones de la fase inicial de su mandato (DW 2020; Cronista 2021c).

La situación regional presentaba una serie de desafíos serios para la política exterior del nuevo presidente argentino. La relación con Brasil, el primer socio comercial de Argentina siendo el destino de más del 15\% de sus exportaciones y el origen de más del $20 \%$ de sus importaciones, se presentó desde un principio como un tema de agenda extremadamente delicado para la diplomacia argentina. Ya durante la campaña presidencial, Jair Bolsonaro advirtió que no quería "otra Venezuela más al Sur del continente," entre otras críticas vertidas a CF y AF, a lo que este último respondió señalando que el presidente brasileño era "un racista, un misógino, un violento" (La Nación 2019; Télam 2019). 
Finalmente, el vicepresidente de Brasil, Hamilton Mourão, asistió a la asunción presidencial de Fernández, ocasión en la que señaló la necesidad de construir relaciones cada vez más "fuertes, maduras y recíprocamente provechosas" (Mourão 2019). Esto marcaría el tono general de las relaciones bilaterales durante todo el 2020, en lo que sería una mezcla de acercamientos impuestos por las circunstancias y críticas a las políticas domésticas e internacionales.

En este marco, la primera reunión bilateral sería recién el 30 de noviembre de 2020, prácticamente un año después de la asunción del presidente argentino. Previamente, las críticas fueron moneda corriente, especialmente en las redes sociales, donde Jair y Eduardo Bolsonaro han hecho referencia en más de una ocasión al gobierno argentino y el estado de su economía, a menudo comparando al país con Cuba o Venezuela, o lamentando la derrota de Mauricio Macri en las elecciones presidenciales de 2019 (J. Bolsonaro 2019; E. Bolsonaro 2020; La Nación 2021). Por su parte, la cercanía y los contactos entre AF y el ex presidente de Brasil Lula Da Silva, era vistos desde Brasilia como una afronta o al menos como intenciones ambiguas acerca de un potencial acercamiento. Además de las críticas generales, también las posiciones encontradas sobre varios temas de agenda generaron el incremento de las fricciones entre ambos gobiernos. En este sentido, podemos identificar cuestiones como la votación para la presidencia del Banco Interamericano de Desarrollo, el acuerdo Mercosur - Unión Europea, o la propia pandemia de COVID-19. La relación con Brasil, de este modo, se ve afectada por dos fuerzas contrapuestas: por un lado, la distancia ideológica con el gobierno de Bolsonaro; por el otro, el rol central que tiene la asociación económica con el país vecino, no sólo para el país en su conjunto, sino para numerosos sectores domésticos que se benefician de ella. Esta diferencia no marcaba una división dentro de la coalición de gobierno, sino una tensión interna entre la ideología y los intereses económicos que cruzaba transversalmente a todos los actores. Esta particular situación limitaba las opciones del gobierno para relacionarse con su principal socio pero no generaba, al menos de forma extendida, diferencias hacia dentro del Frente de Todos.

Las bases de su coalición también mostraban una posición unificada en relación a la situación boliviana, donde el rechazo al gobierno de Añez y el apoyo a Evo Morales eran unánimes. En este caso además la restricción material para el gobierno era prácticamente nula y restringida solamente a la importación de gas: en términos comerciales, Bolivia aparece como un socio menor. Frente a los muy bajos costos potenciales y a los beneficios en términos de apoyo doméstico, el gobierno argentino fue marcadamente más asertivo en la oposición al gobierno boliviano, la crítica al quiebre democrático allí ocurrido, y el apoyo a Evo Morales y el sector político que este representaba, lo que se observa en la gestión del asilo a fines de 2019, las diferentes muestras de apoyo y la propia presencia de AF en la vuelta de Morales a su país en noviembre de 2020 (Télam 2020).

La relación con Venezuela ofrece, por el contrario, un caso claramente diferente a los mencionados. En primer lugar, el caso venezolano representa el caso de 
mayor retroceso democrático y de nivel de ingreso en la región en los últimos 20 años, en una situación marcada por denuncias de graves violaciones de derechos humanos y con una consecuencia de más de 5 millones de emigrantes y refugiados. En segundo lugar, la condena internacional extendida hacia el régimen de Maduro aumentó sustancialmente los costos de demostrar apoyo a su gobierno. En tercero, la posición sobre Venezuela parece interpelar fuertemente a la sociedad argentina y ser un elemento polarizante en el debate político (Sagarzazu y Mouron 2017), donde no sólo la oposición condena severamente al régimen bolivariano, sino que también algunos sectores de la propia coalición gobernante se han manifestado críticos. Esto contrasta con el apoyo que régimen de Maduro aún tiene en otros sectores de la coalición y que limitan el accionar del presidente.

Esta particular situación repercutió en un posicionamiento plagado de marchas, contramarchas y señales ofrecidas en ambas direcciones. En el mes de octubre, las tensiones internas alcanzaron su punto máximo cuando Argentina apoyó el informe presentado por Michele Bachelet en el Consejo de Derechos Humanos de las Naciones Unidas y permaneció dentro del Grupo de Lima, acciones que recibieron fuertes críticas de los sectores más duros del kirchnerismo que componen su coalición. En esta línea, Alicia Castro, integrante del $\mathrm{FpV}$, renunció a su posición de embajadora en Rusia en una carta en la que señalaba que estaba en desacuerdo con la política exterior del gobierno nacional cuyo accionar "no difiere en absoluto de lo que hubiera votado el gobierno de Macri," al tiempo que remarcaba que dentro de su coalición existen dirigentes "opuestos al socialismo venezolano - sin haber pisado nunca Venezuela- y hasta alguno que celebró la proclamación de Guaidó" (Página 12 2020a). Desde el Frente Grande y el Instituto Patria, también cercanos a CF, expresaron de forma pública su malestar en el mismo sentido (Infobae 2020b). Como forma de moderar su posición, el gobierno también estableció que no acompañaría la declaración realizada por el Grupo de Lima, que no reconocería al gobierno de Guaidó y que se opondría a la implementación de sanciones económicas y bloqueos al régimen de Maduro (Infobae 2020d).

Una situación similar se repetiría dos meses más tarde en el seno de la Organización de Estados Americanos (OEA) en la votación en que se buscaba condenar las elecciones realizadas en Venezuela como fraudulentas. El gobierno finalmente se abstuvo aunque su posición no estuvo igual exenta de críticas desde sectores de su propia coalición. De hecho, el propio Diosdado Cabello también atacó a AF llamándolo tibio y remarcando que tanto él como su Canciller atacaban constantemente al gobierno venezolano (Infobae 2020a). La situación parece repetirse de modo similar en el caso de la votación en la OEA sobre las violaciones a los derechos humanos en Nicaragua durante el 2021, frente a la cual el gobierno volvió a abstenerse en una posición que fue blanco de críticas, tanto dentro de la coalición como por parte de la oposición.

En un marco de cierto aislamiento regional, AF buscó potenciar la asociación desde el comienzo mismo de su gobierno con su par mexicano. Además de las 
coincidencias ideológicas y de la posición compartida con respecto a los casos de Venezuela y Bolivia, ambos gobiernos encontraron un nuevo punto de cooperación sobre el cual afianzar el acercamiento: la producción conjunta de las vacunas para el COVID-19 y la importancia de esto para toda la región. En este sentido, Felipe Solá publicó en sus redes sociales que "Para América Latina la vacuna que vamos a producir en la Argentina y México expresa una solidaridad práctica, concreta, sentida e impulsada por el Estado" (Solá 2020). Esta alianza con México, más el posterior acuerdo con Rusia para la compra de la vacuna Sputnik V, permitiría también la búsqueda de una diplomacia regional basada en las vacunas. En diciembre, AF declaró que había conversado con los cancilleres de Uruguay y Bolivia para ayudar a ambos países a conseguir las vacunas provenientes de Rusia, lo que fue desmentido en el primer caso y se materializó en el segundo con un envío de 20 mil vacunas un mes más tarde. Por su parte, en la visita presidencial a México dos meses más tarde, López Obrador afirmó además que su país pudo acceder a la Sputnik V gracias a la intermediación del gobierno argentino (DW 2021).

\section{COMENTARIOS FINALES}

En el futuro inmediato, las tres cuestiones que analizamos aquí, y la política argentina en general, estarán dominadas por las elecciones de medio término. En primer lugar, la pandemia no se ha terminado y seguirá teniendo impactos tanto sanitarios como económicos. En este sentido, la velocidad y efectividad de la campaña de vacunación parece ser la apuesta más fuerte para contrarrestar el descenso de la imagen positiva del gobierno en la opinión pública. Particularmente importante resultará la vacunación si permite al gobierno prescindir de nuevas restricciones a la actividad social y económica.

Segundo, como explicamos anteriormente el gobierno tiene fuertes incentivos para acordar con el Fondo Monetario Internacional antes de las elecciones y poder volver a emitir deuda en el exterior, disminuyendo así las presiones inflacionarias que genera la financiación por emisión. Si esto sucede, será clave en un escenario electoral la forma en que los diferentes miembros de la coalición, especialmente aquellos que han expresado reservas sobre un posible acuerdo, presentan al público las consecuencias económicas y sociales del mismo.

Finalmente, en cuanto a la política exterior, el presidente podría encontrar en esta área una forma de satisfacer demandas de los sectores más duros de su coalición. Si bien analizamos estas tres cuestiones por separado, la vinculación de cuestiones podría ofrecer a Alberto Fernández oportunidades para manejar las tensiones al interior del gobierno. Por ejemplo, concesiones en el área de política exterior podrían funcionar como pagos laterales por políticas menos favorables en otros temas. Hasta que punto esta estrategia es viable dependerá, lógicamente, de cómo esos grupos valoren esas concesiones en relación a lo que pierden en otros ámbitos. De cualquier forma, quedará por verse si los incen- 
tivos electorales para mantener la unidad de la coalición atenuarán las discrepancias o si, por el contrario, algunos de sus miembros intentarán separarse de los aspectos menos exitosos de este primer periodo de gestión.

\section{REFERENCIAS}

Albala, Adrián. 2021. "When Do Coalitions Form Under Presidentialism, and Why Does It Matter? A Configurational Analysis From Latin America." Politics 41(3): 351-370.

Anria, Santiago, y Gabriel Vommaro. 2020. “En Argentina, un 'giro a la derecha' que no fue y el improbable regreso del peronismo de centro-izquierda." Más poder local 40: 6-10.

ANSES. 2020. "Boletín IFEI-2020: Caracterización de la Población Beneficiaria." ANSES: Dirección General de Planeamiento.

Argüello, Jorge. 2019. “Entender el Mundo, Decidir con Autonomía." Le Monde Diplomatique, no. 244.

Axelrod, Robert M. 1970. Conflict of Interest: A Theory of Divergent Goals with Applications to Politics." Chicago: Markham Publishing Company

Biglaiser, Glen, y Ronald J McGauvran. 2021. "The Effects of Debt Restructurings on Income Inequality in the Developing World." European Journal of International Relations. Online first.

Bolsonaro, Eduardo. 2020, 22 de agosto. Twitter. Recuperado el 15 de julio de 2021 de https:/ / twitter.com/BolsonaroSP/status/1297139786914856962.

Bolsonaro, Jair. 2019, 6 de noviembre. Twitter. Recuperado el 15 de julio de 2021 de https:/ / twitter.com/jairbolsonaro/status/1192225819743137792

Borges, André, Mathieu Turgeon, y Adrián Albala. 2020. "Electoral Incentives to Coalition Formation in Multiparty Presidential Systems." Party Politics. Online first https://doi. org $/ 10.1177 / 1354068820953527$

Cheibub, José Antonio, Adam Przeworski, y Sebastian M Saiegh. 2004. "Government Coalitions and Legislative Success Under Presidentialism and Parliamentarism." British Journal of Political Science 34(4): 565-587.

CIDH. 2019a. "CIDH condena el uso excesivo de la fuerza en el contexto de las protestas sociales en Chile, expresa su grave preocupación por el elevado número de denuncias y rechaza toda forma de violencia". Organización de Estados Americanos. Recuperado el 19 de julio de 2021 de http://www.oas.org/es/cidh/prensa/comunicados/2019/317.asp

CIDH. 2019b. "CIDH anuncia Misión de Observación a Ecuador en contexto de las protestas". Organización de Estados Americanos. Recuperado el 19 de julio de 2021 de https: / / www.oas.org/es/cidh/prensa/comunicados/2019/262.asp

CIDH. 2020. "La CIDH condena violaciones a los derechos humanos ocurridas en el contexto de las protestas sociales en Perú, llama a resolver la crisis institucional por vías democráticas, y se pone a disposición del Estado peruano para realizar una visita de trabajo". Organización de Estados Americanos. Recuperado el 19 de julio de 2021 de https:/ / www.oas.org/es/cidh/prensa/comunicados/2020/275.asp

Clerici, Paula Andrea. 2015. "La creciente importancia de las alianzas electorales en un escenario de competencia territorializada: el caso argentino." Revista S.A.A.P 9(2): 313-341.

Corigliano, Francisco. 2011. "La Política Exterior Del Gobierno de Cristina Fernández de Kirchner: Una Típica Política Peronista Del Siglo XXI". Mural Internacional 2(1): 22-27

Cronista 2020a, 11 de noviembre. "Bono IFE ANSeS: Guzmán confirmó que no habrá un cuarto pago del subsidio de \$ 10.000". Recuperado el 15 de julio de 2021 de https://www. cronista.com/economia-politica / Bono-IFE-ANSeS-Guzman-confirmo-que-no-habra-un-cuarto-pago-del-subsidio-de-10-000-20201111-0053.html

Cronista. 2021a, 13 de mayo. "El decálogo del kirchnerismo duro contra Guzmán sumó reclamos y críticas." Recuperado el 15 de julio de 2021 de https://www.cronista.com/ 
economia-politica/el-decalogo-del-kirchnerismo-duro-contra-guzman-sumo-reclamos-y-criticas/

Cronista. 2021b, 13 de mayo. "El Senado aprobó el proyecto para que los DEG del FMI se destinen a la pandemia y no a pagar deuda". Recuperado el 15 de julio de 2021 de https://www.cronista.com/economia-politica/el-senado-trata-hoy-el-proyecto-para-que-los-deg-del-fmi-se-destinen-a-la-pandemia-y-no-a-pagar-deuda /

Cronista. 2021c, 10 de mayo. "Argentina aceptará cambios para 'salvar' el acuerdo Mercosur Unión Europea." Recuperado el 15 de julio de 2021 de https://www.cronista.com/ economia-politica/argentina-aceptara-cambios-para-salvar-el-acuerdo-mercosur-union-europea/

Cruz, Facundo. 2019. Socios pero no tanto: Partidos y coaliciones en la Argentina. 2003- 2015. Vol. 1. Buenos Aires: Eudeba.

Daby, Mariela, y Mason W Moseley. 2021. "Feminist Mobilization and the Abortion Debate in Latin America: Lessons from Argentina." Politics \& Gender 1-35.

DW. 2020, 3 de febrero. "Alberto Fernández insta a materializar acuerdo UE-Mercosur." Recuperado el 15 de julio de 2021 de https://www.dw.com/es/alberto-fern\%C3\%A1ndez-insta-a-materializar-acuerdo-ue-mercosur/a-52247806

DW. 2021, 22 de febrero. "Alberto Fernández llega en visita oficial a México." Recuperado el 15 de julio de 2021 de https://www.dw.com/es/alberto-fern\%C3\%A1ndez-llega-en-visita-oficial-a-m\%C3\%A9xico/a-56655639

El País. 2020a, 7 de febrero. “Alberto Fernández logra el apoyo europeo para renegociar la deuda argentina" Recuperado el 15 de julio de 2021 de https:/ /elpais.com/internacional/2020/02/05/argentina/1580929822_126873.html

El País. 2020b, 17 de diciembre. "Argentina se enreda con la vacuna rusa días antes de la llegada al país de las primeras dosis." Recuperado el 15 de julio de 2021 de https:// elpais.com/sociedad/2020-12-17/argentina-se-enreda-con-la-vacuna-rusa-dias-antes-de-la-llegada-al-pais-de-las-primeras-dosis.html

El País. 2020c, 13 de mayo. "Queremos convertir Argentina en un buen deudor." Recuperado el 15 de julio de 2021 de https://elpais.com/internacional/2020-05-13/queremos-convertir-argentina-en-un- buen-deudor.html

Etchemendy, Sebastián. 2020. "The Politics of Popular Coalitions: Unions and Territorial Social Movements in Post-Neoliberal Latin America (2000-15)." Journal of Latin American Studies 52(1): 157-188.

Fearon, James D. 1998. "Bargaining, Enforcement, and International Cooperation." International Organization 52(2): 269-305.

FMI. 2020. "Comunicado Técnico del Personal del FMI sobre Argentina," Recuperado el 15 de julio de 2021 de https://www.imf.org/es/News/Articles/2020/06/01/pr20228-argentina-imf-staff-technical-statement

Garriga, Ana Carolina, y Juan Negri. 2020. "It's (Almost) Always the Economy: Economic Performance and Political Realignments in Argentina in 2019." Revista de Ciencia Política 40 (2): 137-161.

Gervasoni, Carlos. 2018. “Argentina's Declining Party System: Fragmentation, Denationalization, Factionalization, Personalization, and Increasing Fluidity." En Party Systems in Latin America: Institutionalization, Decay, and Collapse, editado por Scott Mainwaring. Cambridge: Cambridge University Press, 255-290.

INDEC. 2020a. "Estimador Mensual de Actividad Económica - Diciembre de 2020." Cuentas Nacionales 5 (3). Instituto Nacional de Estadística y Censos. Recuperado el 15 de julio de 2021 de https://www.indec.gob.ar/uploads/informesdeprensa/emae_02_21B55CD1AC34.pdf

INDEC. 2020b. "Incidencia de la Pobreza Y la Indigencia en 31 Aglomerados Urbanos - Primer Semestre de 2020." Condiciones de Vida 4 (13). Instituto Nacional de Estadística y Censos. Recuperado el 15 de julio de 2021 de https://www.indec.gob.ar/uploads/informesdeprensa/eph_pobreza_01_200703093514.pdf 
INDEC. 2020c. "Mercado de Trabajo. Tasas e Indicadores socioeconómicos (EPH) - Tercer trimestre de 2020." Trabajo e Ingresos 4 (7). Instituto Nacional de Estadística y Censos. Recuperado el 15 de julio de 2021 de https:/ /www.indec.gob.ar/uploads/informesdeprensa/mercado_trabajo_eph_3trim20E927D146A5.pdf

Infobae. 2020a, 17 de diciembre. "Diosdado Cabello volvió a atacar a Alberto Fernández y exigió la lib- ertad de Milagro Sala: 'Tiene la piel delicadita y le molesta que le digan tibio.'" Recuperado el 15 de julio de 2021 de https://www.infobae.com/america/ venezuela/2020/12/17/diosdado-cabello-volvio-a-atacar-a-alberto-fernandez-y-exigio-la-libertad-de-milagro-sala-tiene-la-piel-delicadita-y-le-molesta-que-le-digan-tibio/

Infobae. 2020b, 6 de octubre. "Fuerte malestar en el kirchnerismo duro por la condena del Gobierno a Venezuela en la ONU." Recuperado el 15 de julio de 2021 de https:/ / www.infobae.com/politica /2020/10/06/ fuerte-malestar-en-el-kirchnerismo-duro-por-la-condena-del-gobierno-a-venezuela-en-la-onu/

Infobae. 2020c, 8 de diciembre. "Juan Grabois: 'Se equivocó compañero Presidente en sacar el IFE.'" Recuperado el 15 de julio de 2021 de https://www.infobae.com/politica/2020/12/08/juan-grabois-se-equivoco-companero-presidente-en-sacar-el-ife/

Infobae. 2020d, 13 de octubre. “La Argentina permanecerá en el Grupo Lima pero rechazará una declaración contra Venezuela". Recuperado el 15 de julio de 2021 de https:/ /www. infobae.com/politica/2020/10/13/la-argentina-permanecera-en-el-grupo-lima-pero-rechazara-una-declaracion-contra-venezuela/

Infobae. 2020e, 7 de setiembre. "Siguen las críticas al impuesto a la riqueza: ahora se sumó una entidad rural con un fuerte comunicado" Recuperado el 15 de julio de 2021 de https: / / www.infobae.com/economia/2020/09/07/siguen-las-criticas-al-impuesto-a-la-riqueza-ahora-se-sumo-una-entidad-rural-con-un-fuerte-comunicado/

Infobae. 2021, 3 de mayo. “En medio de la segunda ola, La Cámpora reclamó el regreso del IFE: 'Es indispensable.'” Recuperado el 15 de julio de 2021 de https:/ / www.infobae.com/ economia/2021/05/03/en-medio-de-la-segunda-ola-la-campora-

Kellam, Marisa. 2017. "Why Pre-Electoral Coalitions in Presidential Systems?" British Journal of Political Science 47(2): 391-411.

La Nación. 2019, 13 de agosto. “Alberto Fernández dijo que Jair Bolsonaro es 'racista, misógino y violento.'” Recuperado el 15 de julio de 2021 de https: / www.lanacion.com.ar/ politica/alberto-fernandez-dijo-jair-bolsonaro-es-racista-nid2277098/

La Nación. 2020a, 3 de noviembre. "Coronavirus en la Argentina: críticas de referentes opositores a la compra de 10 millones de dosis de la vacuna rusa." Recuperado el 15 de julio de 2021 de https://www.lanacion.com.ar/politica/criticas-oposicion-compra-dosis-vacuna-rusa-nid2497888/

La Nación. 2020b, 8 de febrero. "Desde Cuba: Cristina Kirchner le reclamó al FMI una 'quita sustancial' de la deuda". Recuperado el 15 de julio de 2021 de https://www.lanacion. com.ar/politica/sinceramente-marco-su-visita-cuba-enfermedad-su-nid2331925/

La Nación. 2021, 22 de febrero. “'Epidemia de desilusión'. El mensaje en Twitter de Jair Bolsonaro contra el gobierno de Alberto Fernández." Recuperado el 15 de julio de 2021 de https://www.lanacion.com.ar/politica/jair-bolsonaro-alberto-fernandez-twitter-mensaje-coronavirus-nid2609935/

La Política Online. 2020, 19 de mayo. "Moroni ratificó el acuerdo de suspensiones en Mondelez que criticó Máximo." Recuperado el 15 de julio de 2021 de https: / www.lapoliticaonline.com.ar/nota/126691-moroni-ratifico-el-acuerdo-de-suspensiones-en-mondelez-que-critico-maximo/

Laver, Michael, y Kenneth A Shepsle. 1990. "Coalitions and Cabinet Government." American Political Science Review 84(3): 873-890.

Martin, Lanny W, y Georg Vanberg. 2004. "Policing the Bargain: Coalition Government and Parliamentary Scrutiny." American Journal of Political Science 48(1): 13-27.

Martin, Lanny W, y Georg Vanberg. 2008. "Coalition Government and Political Communication." Political Research Quarterly 61(3): 502-516. 
Merke, Federico y Diego Reynoso 2016. “Dimensiones de Política Exterior en América Latina Según Juicio de Expertos". Estudios Internacionales 48(185): 107-130.

Merke, Federico. 2019. "Preferencias, Herencias Y Restricciones: Elementos Para Examinar la Política Exterior Del Frente de Todos." Análisis Carolina 24.

Mourão, Hamilton. 2019, 10 de diciembre. Twitter. Recuperado el 15 de julio de 2021 de https: / / twitter.com/GeneralMourao/status/1204459518442311681

MRECIC. 2020, 12 de diciembre. "Cancillería en Línea." Ministerio de Relaciones Exteriores, Comercio Internacional y Culto de la República Argentina. Recuperado el 15 de julio de 2021 de https://www.cancilleria.gob.ar/userfiles/prensa/newsletter_12_de_diciembre. pdf

Murillo, Maria Victoria, y Rodrigo Zarazaga. 2020. "Argentina: Peronism Returns." Journal of Democracy 31(2): 125-136.

Negretto, Gabriel L. 2006. "Minority Presidents and Democratic Performance in Latin America." Latin American Politics and Society 48(3): 63-92.

Página 12. 2020a, 7 de octubre. "La carta completa de renuncia de Alicia Castro a la embajada de Rusia" Recuperado el 15 de julio de 2021 de https://www.pagina12.com. ar/297464-la-carta-completa-de-renuncia-de-alicia-castro-a-la-embajada

Panizza, Ugo, Federico Sturzenegger, y Jeromin Zettelmeyer. 2009. "The Economics and Law of Sovereign Debt and Default." Journal of economic literature 47(3): 651-98.

Perfil. 2020a, 18 de noviembre. "La crítica de un diputado del Frente de Todos: 'El gobierno se equivocó al sacar el cuarto IFE.'” Recuperado el 15 de julio de 2021 de https: / /www. perfil.com/noticias/politica/critica-diputado-frente-de-todos-juan-carlos-alderete-gobierno-se-equivoco-al-sacar-el-cuarto-ife.phtml

Perfil. 2020b, 6 de febrero. “Trump recibió a Jorge Argüello y prometió apoyo: ‘Dígale al presidente Fernández que cuenta conmigo.'” Recuperado el 15 de julio de 2021 de https: / / www. perfil.com/noticias/actualidad/donald-trump-recibio-jorge-arguello-embajador-argentino-prometio-apoyo-renegociacion-deuda.phtml

Perfil. 2021, 25 de marzo. "Bonafini: 'Señor Presidente no se acostumbre a mentir, con Guzmán nos engañaron todo el tiempo.'” Recuperado el 15 de julio de 2021 de https:/ / www.perfil.com/noticias/actualidad/bonafini-senor-presidente-no-se-acostumbre-a-mentir-con-guzman-nos-enganaron-todo-el-tiempo.phtml

Poliarquía \& Wilson Center. 2019, 5 de diciembre. "Argentina Pulse 5. La Percepción de Los Argentinos en Relación Al Orden Mundial, La Política Exterior Y Los Problemas Globales." Recuperado el 15 de julio de 2021 de http://poliarquia.com/wp-content/ uploads/2019/12/Arg-Pulse_INFORME-5-ESP.pdf

Poliarquía \& Wilson Center. 2020, 20 de abril. "Argentina Pulse 6. La Percepción de Los Argentinos en Relación Al Orden Mundial, La Política Exterior Y Los Problemas Globales" (April 20). Recuperado el 15 de julio de 2021 de https: / / www.wilsoncenter.org/sites/default/ files/media/uploads/documents/Arg\%20Pulse_INFORME\%206\%20\%28ESP\%29.pdf

Riker, William H. 1962. The Theory of Political Coalitions. New Haven: Yale University Press.

Sagarzazu, Iñaki, y Fernando Mouron. 2017. "Hugo Chavez's Polarizing Legacy: Chavismo, Media, and Public Opinion in Argentina's Domestic Politics." Revista de Ciencia Politica 37(1): 147-175.

Schraff, Dominik. 2020. "Political Trust During the COVID-19 Pandemic: Rally Around the Flag or Lockdown Effects?" European Journal of Political Research. Online First https:/ /doi. org/10.1111/1475-6765.12425

Secretaría de Finanzas. 2019. Deuda de la Administración Central - IV Trimestre 2019. Recuperado el 20 de marzo de 2021 de https://www.argentina.gob.ar/sites/default/files/ presentacion_grafica_de_la_deuda_31-12-2019_0.pdf

Solá, Felipe. 2020, 28 de agosto. Twitter. Recuperado el 15 de julio de 2021 de https:/ / twitter.com/felipe_sola/status/1299451797359210496?lang=es

Télam. 2019, 3 de mayo. "Bolsonaro dijo que 'el mundo entero' no quiere otra Venezuela en Argentina." Recuperado el 15 de julio de 2021 de https://www.telam.com.ar/notas/201905/354927-bolsonaro-argentina-venezuela-macri-cristina-kirchner-brasil.html 
Télam. 2020, 20 de noviembre. “El Presidente despidió a Evo Morales y revindicó ‘la Patria Grande que quiere crecer.'” Recuperado el 15 de julio de 2021 de https: / / www.telam. com.ar/notas/202011/533550-fernandez-quiaca-evo-morales.html

Tomz, Michael. 2007. Reputation and International Cooperation: Sovereign Debt Across Three Centuries. Princeton: Princeton University Press.

Universidad de San Andrés. 2020a. “Encuesta de Satisfacción Política y Opinión Pública

Diciembre 2020." Recuperado el 25 de marzo de 2021 de https:/ /udesa.edu.ar/sites/default/ files/22._udesa_espop_diciembre_2020.pdf

Universidad de San Andrés. 2020b. “Encuesta de Satisfacción Política y Opinión Pública

Octubre 2020." Recuperado el 25 de marzo de 2021 de https://udesa.edu.ar/sites/default/ files/21._udesa_espop_octubre_2020_0.pdf

Universidad de San Andrés. 2021. “Encuesta de Satisfacción Política y Opinión Pública - Especial Vuelta a Clases, Marzo de 2021." Recuperado el 25 de marzo de 2021 de https:/ / udesa.edu.ar/sites/default/files/udesa_espop_especial_vuelta_a_clases.pdf

Universidad Torcuato Di Tella. 2021. Índice de Confianza en el Gobierno. Recuperado el 23 de marzo de 2021 de $h t t p s: / / w w w . u t d t . e d u / i c g$

Zarazaga, Rodrigo. 2019. “'Todos Unidos Triunfaremos...': Nuevas Dificultades Para la Unidad Electoral Peronista." Revista SAAP: Sociedad Argentina de Análisis Político 13(1): 11-42

Recibido: 30 de marzo de 2021

Aceptado: 8 de julio de 2021

Florencia Montal es Profesora Investigadora Asistente en el Departamento de Ciencia Política y Estudios Internacionales de la Universidad Torcuato Di Tella en Buenos Aires, Argentina. Tiene un doctorado en Ciencia Política por la Universidad de Minnesota y su trabajo se enfoca en la intersección entre el derecho internacional, la diplomacia y la política doméstica, principalmente en las áreas de los derechos humanos, derecho penal internacional y derecho internacional de inversiones. Email: fmontal@utdt.edu.

Patricio Yamin es Profesor de Cátedra en el Departamento de Ciencias Sociales de la Universidad de San Andrés en Buenos Aires, Argentina. Es Candidato a Doctor en Estudios Internacionales en la Universidad Torcuato Di Tella y su trabajo se enfoca prin- cipalmente en el estudio de la política doméstica e internacional del cambio climático y el rol de las coaliciones domésticas en la formulación de la política exterior. Email: pyaminvazquez@udesa.edu.ar. 
